\title{
Erratum: Frequency effects on the scale and behavior of acoustic streaming \\ [Phys. Rev. E 89, 013203 (2014)]
}

Michael B. Dentry, Leslie Y. Yeo, and James R. Friend

(Received 23 September 2016; published 1 November 2016)

DOI: 10.1103/PhysRevE.94.059901

A typographic error is present in Eq. (4) of the original paper. The Rayleigh wave velocity $V_{R}$ should instead be the speed of sound in the liquid $c$ as shown below:

Original form (with the error)

$$
P=\int_{0}^{\infty} \rho w V_{R} \dot{\eta}^{2} \mathrm{~d} y,
$$

corrected form

$$
P=\int_{0}^{\infty} \rho w c \dot{\eta}^{2} d y,
$$

This correction only has a minor effect on the results reported in the paper because the scaling is identical and $V_{R}$ and $c$ are on the same order of magnitude. 